\title{
Cooperative effects in the nonlinearly driven spin-boson system
}

\author{
Milena Grifoni \\ Institut für Physik, Universität Augsburg, Memminger Straße 6, D-86135 Augsburg, Germany \\ Maura Sassetti \\ Istituto di Fisica Ingegneria, Consorzio INFM, Università di Genova, I-16146 Genova, Italy \\ Peter Hänggi \\ Institut für Physik, Universität Augsburg, Memminger Straße 6, D-86135 Augsburg, Germany \\ Ulrich Weiss \\ Institut für Theoretische Physik, Universität Stuttgart, D-70550 Stuttgart, Germany
}

(Received 3 May 1995)

\begin{abstract}
We study the quantum dynamics of a periodically driven biased two-state system which additionally is subject to either Ohmic or to frequency-dependent damping. Within the noninteracting-blip approximation for the stochastic forces, the solution is given in terms of a series which is defined by recursion relations. Avoiding rotating wave and Markovian approximation, we deduce the solution in closed form for the important cases of high- and low-frequency driving. For low-frequency driving, the dynamics is governed by a rate equation with a time-dependent rate and with a time-dependent adiabatic equilibrium state. In the high-frequency case, we find novel cooperative effects, such as resonances in the spectral distribution near fractional values of the static detuning energy.
\end{abstract}

PACS number(s): 05.30. $-\mathrm{d}, 05.40 .+\mathrm{j}, 33.80 . \mathrm{Be}, 62.65 .+\mathrm{k}$

\section{INTRODUCTION}

Often in nature, a quantum-mechanical system with few relevant degrees of freedom is coupled to the surrounding medium and is in addition subject to external time-dependent forces. Consider now the simplest case in which the system is a two-state or two-level system (TLS) and the environment is modeled by a bath of harmonic oscillators with bilinear coupling to the system $[1,2]$. The archetype model we then have is the driven spin-boson model [3]. There has been increasing interest in the behaviors of such systems due to the diversity of physical realizations, as well as to some advances in the theory in recent years and to the interesting phenomena observed. The driven spin-boson model can be applied to describe, e.g., ac-driven superconducting quantum interference devices [4], laser-induced isomerization of bistable molecules $[5,6]$, laser-induced localization of electrons in semiconductor double-well quantum structures [7], or paraelectric resonances [8].

A main theme of driven dissipative bistable systems is the reduction of the coherent tunneling motion by incoherent processes, which are induced by the variation of the tunneling splitting due to fluctuating and driving forces. The frictional influences can lead to qualitative changes in the behaviors. Quite generally, the stochastic force results in a reduction of the tunneling process $[2,9,10]$, and may even lead to a fluctuationinduced transition to self-trapping at zero temperature [11]. Drastic changes do also occur by strong external driving. Quenching of quantum tunneling may be induced by external periodic driving in some regions of the parameter space [12-14]. The effects of driving and dissi- pation have been investigated numerically by Dittrich et al. [15]. They studied the influence of a monochromatic field on the coherent tunneling motion of a symmetric bistable system. The transition from coherent to incoherent dynamics for a symmetric two-state system has also been studied analytically by Dakhnovskii [16] for a high-frequency monochromatic field. However, in Ref. [16] only the transient dynamics of the driven TLS and not the time-periodic state reached at long times is considered.

First of all, it is clear that the often used linearresponse approximation is only meaningful when the driving is sufficiently weak. In previous work [3], we investigated the nonlinear response to a time-periodic force of an asymmetric two-state system embedded in an Ohmic medium. There, we focused the attention on nonlinear driving at low frequencies. Parts of that study were then successfully used to explain recent anomalous behaviors found in experiments on acoustic properties of amorphous metals [17]. These experiments [18, 19] showed strong deviations from the predictions of the standard tunneling model [20] both in sound velocity and in internal friction at very low temperatures and frequencies. Further, most of the data on the acoustic properties in Ref. [19] show amplitude-dependent effects. This indicates that the strain fields in the vibrating reed or vibrating wire experiments are so strong that the linearresponse regime is exceeded.

Cooperative effects of the interplay of noise and periodic perturbations in classical bistable systems have been popularized as "stochastic resonance." In these systems, the response to external systematic driving is enhanced by stochastic forces [21-23]. Recently, it has been 
shown by Löfsted and Coppersmith [24] that in asymmetric bistable quantum systems a similar phenomenon may occur. In these studies, restriction was made to low-frequency driving and to a Markovian description of the TLS dynamics. Against this background, further extension of the theoretical description that cover a wider range of frequencies and amplitudes of the driving force seems important and timely.

In this work, we generalize the results of Ref. [3] in several directions. The exact formal solution of the periodically driven dynamics, previously discussed for Ohmic viscosity, is extended to cover also frequency-dependent damping. For the driven spin-boson problem, a major difficulty arises as follows. Added to the correlations induced by the stochastic force, there are correlations between all the transitions the system makes, which are induced by the driving force. We present a solution in terms of recursion relations for the time-periodic state reached at long times, as well as for the transient behavior, without approximations regarding the driving force and within the noninteracting-blip approximation (NIBA) for the stochastic force. Analytic solutions in closed form for arbitrary strength of the driving amplitude are given in the two limiting cases of "slow" and "fast" driving. We also discuss the linear-response limit for arbitrary frequencies of the periodic force. For lowfrequency driving and in the damping regime where incoherent transitions prevail, the dynamics is described by a rate equation with a time-dependent transition rate. At high frequencies, there are selection rules that enhance or suppress Fourier components of the time-periodic asymptotic state. This leads to a rich structure in the spectral distribution. For the ubiquitous case of Ohmic dissipation, resonance phenomena for driving frequencies near fractional values of the intrinsic static detuning energy of the TLS are found.

The outline of the paper is as follows. In Sec. II, we briefly describe the driven spin-boson model. Section III covers our main results. Starting out from the exact formal solution, we deduce within the NIBA an iterative scheme allowing for the systematic calculation of the dynamics for any strength and frequency of the driving force. In Secs. IV and V, we restrict the attention to driving at high and low frequency, respectively. Finally, in Sec. VI, we summarize the findings and draw some conclusions.

\section{THE DRIVEN SPIN-BOSON MODEL}

A great variety of physical and chemical systems is characterized by a generalized coordinate with which is associated an effective potential energy function with two separated minima $[9,10]$. Consider a slightly asymmetric double well with barrier height $V_{0}$, separation $\hbar \omega_{0}$ of the first excited state from the ground state in either well, and with a "detuning" energy $\hbar \epsilon_{0}$ between the ground states in the two wells. Then, whenever $V_{0} \gg \hbar \omega_{0} \gg \hbar \epsilon_{0}, k T$, where $T$ denotes the temperature, the system will be effectively restricted to the twodimensional Hilbert space spanned by the two ground states. Taking into account the possibility of tunneling between the two wells, the TLS is then governed by the pseudospin Hamiltonian

$$
H_{\mathrm{TLS}}=-(\hbar \Delta / 2) \sigma_{x}-\left(\hbar \epsilon_{0} / 2\right) \sigma_{z} .
$$

Here the $\sigma$ 's are Pauli matrices and the basis states $|r\rangle$ (right) and $|l\rangle$ (left) are eigenstates of $\sigma_{z}$ with eigenvalues +1 and -1 , respectively. The tunneling splitting energy of the symmetric TLS is given by $\hbar \Delta$. Here we consider linear couplings to the heat bath that are sensitive to the value of $\sigma_{z}$. To be definite, we choose $H_{I}=-q \sum_{\alpha} c_{\alpha} x_{\alpha}$, where $q=\sigma_{z} a / 2$ and $a$ denotes a characteristic length (usually the spatial distance of the two localized states). The set $\left\{x_{\alpha}\right\}$ refers to the bath coordinates. Then, by representing the reservoir by an ensemble of harmonic oscillators, we end up with the spin-boson Hamiltonian [2]

$$
H_{\mathrm{SB}}=H_{\mathrm{TLS}}+\frac{1}{2} \sum_{\alpha}\left(\frac{p_{\alpha}^{2}}{m_{\alpha}}+m_{\alpha} \omega_{\alpha}^{2} x_{\alpha}^{2}-c_{\alpha} x_{\alpha} a \sigma_{z}\right) .
$$

To make the model complete, we allow for an externally applied time-periodic force $\hbar \hat{\epsilon} f(t) / a$ with amplitude $\hbar \hat{\epsilon} / a$ and period $2 \pi / \omega, f(t)=f(t+2 \pi / \omega)$. Then, the Hamiltonian of the driven spin-boson system that we shall study is

$$
H=H_{\mathrm{SB}}-\hbar \hat{\epsilon} f(t) \sigma_{z} / 2 .
$$

As far as the properties of the TLS are concerned, the coupling constants $c_{\alpha}$ and the parameters of the bath are of importance only via the spectral density of the coupling

$$
J(\omega)=\frac{\pi}{2} \sum_{\alpha} \frac{c_{\alpha}^{2}}{m_{\alpha} \omega_{\alpha}} \delta\left(\omega-\omega_{\alpha}\right), \quad \omega>0 .
$$

There are three bias-influencing forces: an intrinsic constant force $\hbar \epsilon_{0} / a$, an externally applied timedependent force $\hbar \hat{\epsilon} f(t) / a$, and a fluctuating force $\zeta(t)=$ $\sum_{\alpha} c_{\alpha} x_{\alpha}(t)$. For a harmonic bath, the fluctuating force obeys Gaussian statistics, and is fully characterized by $\langle\zeta(t)\rangle_{\beta}=0$ and by the force autocorrelation function in thermal equilibrium $[2,9]$

$$
\langle\zeta(t) \zeta(0)\rangle_{\beta}=\frac{\hbar}{\pi} \int_{0}^{\infty} d \omega J(\omega) \frac{\cosh [\omega(\hbar \beta / 2-i t)]}{\sinh (\omega \hbar \beta / 2)},
$$

where $\beta \equiv 1 / k T$, and \langle\rangle$_{\beta}$ denotes thermal average over the bath degrees of freedom. Since we wish the environment to constitute a proper heat bath, we shall consider $J(\omega)$ as continuous henceforth. In the classical description, the spectral density $J(\omega)$ is related to the frequency-dependent friction [1]. At low temperatures, the low-frequency dependence of $J(\omega)$ is crucial for the qualitative physics, while the high-frequency modes essentially cause an adiabatic renormalization of the bare tunneling splitting $\Delta[2,9,10]$. Here we shall consider a class of models with a power-law form at low frequencies and a smooth exponential cutoff at the scale $\omega_{c}$. We 
make the specific choice

$$
J(\omega)=\left(2 \pi \hbar \alpha_{s} / a^{2}\right) \omega^{s} \tilde{\omega}^{1-s} e^{-\omega / \omega_{c}},
$$

where $\alpha_{s}$ is a dimensionless coupling parameter, and $\alpha_{s} \tilde{\omega}^{1-s}$ is the dimensional coupling strength for $s \neq 1$. In what follows, we shall assume that the cutoff frequency $\omega_{c}$ in the environmental modes is the highest frequency of the problem. The case $s=1$ is known to describe Ohmic friction in the classical limit. As shown by Kondo and others (see, e.g., Ref.[25]), the frictional influences of the electron-hole excitations near to the Fermi surface of a Fermi liquid are essentially equivalent to that of a bath of bosons with spectral density $J(\omega) \propto \omega$. Ohmic dissipation is widespread in many physical and chemical situations. For instance in metallic glasses at low temperatures, the most relevant coupling of TLS's is the coupling to conduction electrons [20]. In difference to this, for defect tunneling in insulating solids, super-Ohmic friction with $s=3$ or $s=5$ (depending on the lattice symmetry) applies [26]. Super-Ohmic friction also determines the acoustic dispersive and absorptive properties of dielectric glasses [27].

\section{THE DYNAMICS UNDER DRIVING}

The central quantity we are interested in is the expectation value $\left\langle\sigma_{z}(t)\right\rangle$ of the TLS for the case that the TLS-plus-bath complex is described initially, say at time $t_{0}=0$, by a density matrix in factorized form. To be definite, we assume that the TLS starts out from the state $|r\rangle$ while the bath is in thermal equilibrium. Then, the expectation value of $\sigma_{z}$ is given by

$$
\left\langle\sigma_{z}(t)\right\rangle \equiv P(t)=P(+, t ;+, 0)-P(-, t ;+, 0),
$$

where $P\left(\sigma, t ; \sigma^{\prime}, t^{\prime}\right)$ is the joint probability for finding the system in the state $\sigma$ at time $t$, which developed from the state $\sigma^{\prime}$ at time $t^{\prime}$. For the Ohmic case, an exact formal expression for the joint probability has been derived in Ref. [3]. Before embarking on the explicit evaluation of the resulting formidable expression, we first present the generalization to arbitrary spectral densities of the coupling.

\section{A. Exact formal solution}

According to Feynman-Vernon theory [28], the joint probability of the reduced system is given by the double path integral expression

$P\left(\sigma, t ; \sigma^{\prime}, 0\right)=\int \mathcal{D} q \int \mathcal{D} q^{\prime} \mathcal{A}[q] \mathcal{B}[q] \mathcal{A}^{*}\left[q^{\prime}\right] \mathcal{B}^{*}\left[q^{\prime}\right] \mathcal{F}\left[q, q^{\prime}\right]$

Here, the sum is over all paths $q\left(t^{\prime}\right), q^{\prime}\left(t^{\prime}\right)$ which jump back and forth between the values $\pm a / 2$, with constraints $q(0)=q^{\prime}(0)=\sigma^{\prime} a / 2, q(t)=q^{\prime}(t)=\sigma a / 2$. The quantity $\mathcal{A}[q]$ is the probability amplitude of the TLS to follow the path $q\left(t^{\prime}\right)$ in the absence of both driving and stochastic forces. The phase factor

$$
\mathcal{B}[q]=\exp \left\{i \int_{0}^{t} d t^{\prime}\left(\epsilon_{0}+\hat{\epsilon} f\left(t^{\prime}\right)\right) q\left(t^{\prime}\right) / a\right\}
$$

accounts for the history of the systematic biasing forces, and $\mathcal{F}\left[q, q^{\prime}\right]$ is the Feynman-Vernon influence functional encompassing all influences of the stochastic forces. For Gaussian statistics, it takes the form

$$
\begin{aligned}
\mathcal{F}\left[q, q^{\prime}\right]= & \exp \left\{-\int_{0}^{t} d t^{\prime} \int_{0}^{t^{\prime}} d t^{\prime \prime}\left[q\left(t^{\prime}\right)-q^{\prime}\left(t^{\prime}\right)\right]\right. \\
& \left.\times\left[K\left(t^{\prime}-t^{\prime \prime}\right) q\left(t^{\prime \prime}\right)-K^{*}\left(t^{\prime}-t^{\prime \prime}\right) q^{\prime}\left(t^{\prime \prime}\right)\right]\right\}
\end{aligned}
$$

where $K(t) \equiv\langle\zeta(t) \zeta(0)\rangle_{\beta}$ is the force autocorrelation function given previously in (2.4). It is convenient to switch to the symmetric and antisymmetric coordinates,

$$
\eta\left(t^{\prime}\right)=\frac{1}{a}\left[q\left(t^{\prime}\right)+q^{\prime}\left(t^{\prime}\right)\right] ; \quad \xi\left(t^{\prime}\right)=\frac{1}{a}\left[q\left(t^{\prime}\right)-q^{\prime}\left(t^{\prime}\right)\right]
$$

measuring quasiclassical propagation and excursions away from the diagonal, respectively. Now, a two-state system starting out from a diagonal state of the density matrix is again in a diagonal state after any even number of transitions, and in an off-diagonal state after any odd number of transitions. Since the transitions of the system are sudden, a general path with $2 n$ flips at times $t_{j}(j=1,2, \ldots, 2 n)$ is parametrized by

$$
\begin{aligned}
\xi_{n}\left(t^{\prime}\right) & =\sum_{j=1}^{n} \xi_{j}\left[\theta\left(t^{\prime}-t_{2 j-1}\right)-\theta\left(t^{\prime}-t_{2 j}\right)\right], \\
\eta_{n}\left(t^{\prime}\right) & =\sum_{j=0}^{n} \eta_{j}\left[\theta\left(t^{\prime}-t_{2 j}\right)-\theta\left(t^{\prime}-t_{2 j+1}\right)\right],
\end{aligned}
$$

where $t_{0} \equiv 0, t_{2 n+1} \equiv t$, and where $\theta(t)$ is the unit step function. The labels $\xi_{j}= \pm 1$ and $\eta_{j}= \pm 1$ mark the two off-diagonal and diagonal states of the density matrix, respectively. The periods $t_{2 j}<t^{\prime}<t_{2 j+1}$, in which the system is in a diagonal state, are usually referred to as sojourns, and the periods $t_{2 j-1}<t^{\prime}<t_{2 j}$, in which the system stays off-diagonal, are called blips (cf. Refs. $[2,9])$. Now, the sum over the system's past histories is represented (i) by the sum over all numbers $n$ of flip pairs, (ii) by the time-ordered integrations over the $2 n$ flip times $\left\{t_{j}\right\}$ within the given interval, and (iii) by the sum over all arrangements $\left\{\xi_{j}\right\}$ and $\left\{\eta_{j}\right\}$ of the possible values \pm 1 of the individual $\xi_{j}$ and $\eta_{j}$. The amplitude per unit time to switch from a diagonal to an off-diagonal state, or vice versa, is $\pm i \Delta / 2$, and the amplitude to stay in a sojourn is unity. Furthermore, there is a bias phase factor $\exp \left(i \Phi_{n}\right)$. The phase $\Phi_{n}$ receives contributions from the static bias and from the time-dependent strain field. It is given by

$\Phi_{n}=\sum_{j=1}^{n} \xi_{j}\left[\epsilon_{0}\left(t_{2 j}-t_{2 j-1}\right)+g\left(t_{2 j}\right)-g\left(t_{2 j-1}\right)\right]$.

The external driving is in the function $g(t)$. We have

$$
g(t)=\hat{\epsilon} \int_{0}^{t} d t^{\prime} f\left(t^{\prime}\right)
$$


Finally, the influence functional for $n$ flip pairs reads

$$
\begin{aligned}
& \mathcal{F}_{n}=Q_{n}\left(\left\{\xi_{j}\right\}\right) H_{n}\left(\left\{\xi_{j}\right\},\left\{\eta_{j}\right\}\right) \\
& Q_{n}=\exp \left(-\sum_{j=1}^{n} S_{2 j, 2 j-1}-\sum_{j=2}^{n} \sum_{k=1}^{j-1} \xi_{j} \xi_{k} \Lambda_{j, k}\right) \\
& H_{n}=\exp \left(i \sum_{j=1}^{n} \xi_{j} \sum_{k=0}^{j-1} \eta_{k} X_{j, k}\right) .
\end{aligned}
$$

The real-valued function $Q_{n}$ encompasses the selfinteractions $\left\{S_{2 j, 2 j-1}\right\}$ and the interblip interactions $\left\{\Lambda_{j, k}\right\}$ of the $n$ blips. The phase factor $H_{n}$ describes interactions of each blip with all preceding sojourns. The interactions are given by the expressions

$$
\begin{aligned}
& S_{j, k}=S\left(t_{j}-t_{k}\right) ; \quad R_{j, k}=R\left(t_{j}-t_{k}\right) \\
& \Lambda_{j, k}=S_{2 j, 2 k-1}+S_{2 j-1,2 k}-S_{2 j, 2 k}-S_{2 j-1,2 k-1} \\
& X_{j, k}=R_{2 j, 2 k+1}+R_{2 j-1,2 k}-R_{2 j, 2 k}-R_{2 j-1,2 k+1}
\end{aligned}
$$

where the functions $S(\tau)$ and $R(\tau)$ are the real and imaginary part of the second integral of the force autocorrelation function $K(\tau)$,

$$
\begin{aligned}
& S(\tau)=\frac{a^{2}}{\pi \hbar} \int_{0}^{\infty} d \omega \frac{J(\omega)}{\omega^{2}}(1-\cos \omega \tau) \operatorname{coth}\left(\frac{\hbar \beta \omega}{2}\right), \\
& R(\tau)=\frac{a^{2}}{\pi \hbar} \int_{0}^{\infty} d \omega \frac{J(\omega)}{\omega^{2}} \sin \omega \tau .
\end{aligned}
$$

Piecing together all factors and all possible arrangements of blips and sojourns, the joint probability emerges as the series

$$
\begin{aligned}
P\left(\sigma, t ; \sigma^{\prime}, 0\right)= & \delta_{\sigma, \sigma^{\prime}}+\sigma \sigma^{\prime} \sum_{n=1}^{\infty}\left(-\frac{\Delta^{2}}{4}\right)^{n} \\
& \times \int_{0}^{t} \mathcal{D}_{n}\left\{t_{j}\right\} \sum_{\left\{\xi_{j}\right\}} Q_{n} e^{i \Phi_{n}} \sum_{\left\{\eta_{j}\right\}^{\prime}} H_{n} .
\end{aligned}
$$

The prime in $\left\{\eta_{j}\right\}^{\prime}$ indicates that the outer sojourns are chosen according to the boundary conditions, i.e., $\eta_{0}=\sigma^{\prime}$ and $\eta_{n}=\sigma$, and we used the shorthand symbol

$$
\int_{0}^{t} \mathcal{D}_{n}\left\{t_{j}\right\} \cdots:=\int_{0}^{t} d t_{2 n} \int_{0}^{t_{2 n}} d t_{2 n-1} \cdots \int_{0}^{t_{2}} d t_{1} \cdots .
$$

Expression (3.11) represents an expansion in even numbers of transitions between the four states of the density matrix. The transitions occur at times $\left\{t_{j}\right\}$, and the sum over $\left\{\xi_{j}\right\}$ and $\left\{\eta_{j}\right\}^{\prime}$ takes into account all possible intermediate states for the $2 n$ transitions. It is straightforward to perform the summation over the inner $\eta_{j}$, and we finally obtain

$$
\begin{aligned}
P(t)= & 1+\sum_{n=1}^{\infty}\left(-\frac{\Delta^{2}}{2}\right)^{n} \int_{0}^{t} \mathcal{D}_{n}\left\{t_{j}\right\} \\
& \times \sum_{\left\{\xi_{j}\right\}} Q_{n} \cos \left(\Phi_{n}+\varphi_{0, n}\right) \prod_{k=1}^{n-1} \cos \varphi_{k, n}
\end{aligned}
$$

where

$$
\varphi_{k, n}=\sum_{j=k+1}^{n} \xi_{j} X_{j, k}
$$

The expression (3.13) is the exact formal solution for the evolution of $P(t)$ under time-dependent external forces. The complete history of the biasing forces is in the phase $\Phi_{n}$ given in (3.6). It is convenient to decompose $P(t)$ as

$$
P(t)=P^{(a)}(t)+P^{(s)}(t),
$$

where $P^{(a)}(t)$ and $P^{(s)}(t)$ are odd and even functions of the bias $\varepsilon(t):=\epsilon_{0}+\hat{c} f(t)$. As we shall see, the symmetric part $P^{(s)}(t)$ is damped away. Only $P^{(a)}(t)$ is relevant in the asymptotic regime.

To study the frequency-dependent response, it is convenient to switch to the Laplace transform $\widehat{P}(\lambda)=$ $\int_{0}^{\infty} d t P(t) e^{-\lambda t}$. To this, we replace the integrations over the flip times by integrations over the blip lengths $\tau_{j}$ and sojourn lengths $\rho_{j}$,

$\tau_{j}:=t_{2 j}-t_{2 j-1} ; \rho_{j}:=t_{2 j-1}-t_{2 j-2} ;(j=1,2, \ldots, n)$.

Upon introducing the compact notation

$$
\begin{aligned}
\int_{0}^{\infty} \tilde{\mathcal{D}}_{n}(\lambda) \cdots \\
\quad:=\prod_{i=1}^{n}\left(\int_{0}^{\infty} d \tau_{i} e^{-\lambda \tau_{i}} \int_{0}^{\infty} d \rho_{i} e^{-\lambda \rho_{i}}\right) \cdots,
\end{aligned}
$$

the Laplace transform of $P(t)$ takes the form

$$
\begin{aligned}
\widehat{P}(\lambda)= & \frac{1}{\lambda}+\frac{1}{\lambda} \sum_{n=1}^{\infty}\left(-\frac{\Delta^{2}}{2}\right)^{n} \int_{0}^{\infty} \widetilde{\mathcal{D}}_{n}(\lambda) \\
& \times \sum_{\left\{\xi_{j}\right\}} Q_{n} \cos \left(\Phi_{n}+\varphi_{0, n}\right) \prod_{k=1}^{n-1} \cos \varphi_{k, n} .
\end{aligned}
$$

For later convenience, we rewrite the phase $\Phi_{n}$ defined in (3.6) in the form

$$
\Phi_{n}=\sum_{j=1}^{n} \xi_{j} \phi_{j} ; \quad \phi_{j}=\phi_{j}^{(0)}+\phi_{j}^{(1)}
$$

where the contributions related to the $j$ th blip read

$$
\begin{aligned}
& \phi_{j}^{(0)}=\epsilon_{0} \tau_{j} \\
& \phi_{j}^{(1)}=g\left(\sum_{l=1}^{j}\left(\tau_{l}+s_{l}\right)\right)-g\left(s_{j}+\sum_{l=1}^{j-1}\left(\tau_{l}+s_{l}\right)\right)
\end{aligned}
$$

From this we see that the phase $\phi_{j}^{(1)}$, which is associated with the time-dependent strain field, depends on all the preceding blip and sojourn lengths. This peculiar feature makes an exact calculation of Eq. (3.18) extremely difficult. 


\section{B. Recursive solution}

Despite its complicated appearance, the expression (3.13) for $P(t)$ can be summed analytically in some regions of the parameter space. In the static case, the major difficulty for an analytic resummation arises from the correlations $\Lambda_{j, k}$ and $X_{j, k}$ in the factors $Q_{n}$ and $H_{n}$ in (3.8), respectively. In the presence of time-dependent driving, the situation is even more intricate since we also have to take into account the correlations of the blip and sojourn lengths in the phase $\Phi_{n}$. Here we shall take advantage of a well-known approximation for the stochastic correlations, the justification of which has been abundantly discussed in the literature (see, e.g., Refs. [2] and [9]), and which is known as the NIBA. In the NIBA, it is assumed (and it has been shown self-consistently) that the average blip length $\langle\tau\rangle$ is sufficiently small against the average sojourn length $\langle\rho\rangle$,

$$
\langle\tau\rangle \ll\langle\rho\rangle,
$$

so that the bath-induced interblip and blip-sojourn correlations are negligible.

For Ohmic dissipation $\left[s=1\right.$ in $\left.(2.5), \alpha_{1}:=\alpha\right]$ and times $\tau \gg 1 / \omega_{c}$, the complex-valued interaction (3.10) is given by

$$
S(\tau)+i R(\tau)=\alpha\left\{2 \ln \left[\frac{\hbar \beta \omega_{c}}{\pi} \sinh \left(\frac{\pi \tau}{\hbar \beta}\right)\right]+i \pi\right\}
$$

The intrablip interactions $S_{2 j, 2 j-1}$ in the function $Q_{n}$ in (3.8) lead to exponential suppression $\propto \exp \left(-2 \alpha \pi \tau_{j} / \hbar \beta\right)$ of long blips while long sojourns are not suppressed by such factor. One finds that for "strong" damping the NIBA is justified in the entire parameter region. For "weak" damping, the interaction (3.22) is still effective in reducing the blip length if the temperature is high enough. In particular, under the condition $\hbar \beta \Delta \ll 1$, we have $\langle\tau\rangle / \hbar \beta \gg 1$, which allows for the approximation

$$
S(\tau)=2 \alpha\left(\ln \frac{\hbar \beta \omega_{c}}{2 \pi}+\frac{\pi \tau}{\hbar \beta}\right)
$$

With this form, the interblip correlations $\Lambda_{j, k}$ are zero, and the NIBA becomes exact.

In the super-Ohmic case, the function $S(\tau)$ behaves at long times and finite temperatures as $\tau^{2-s}$. Hence the function $S(\tau)$ increases with $\tau$ for long times when $s<2$ and therefore long blips are suppressed by the intrablip interaction. For $s>2$, the function $S(\tau)$ is no longer effective in suppressing long blip lengths. Nevertheless, the NIBA can still be justified for arbitrary spectral densities as long as the system exhibits overdamped exponential relaxation (see also the discussion in Sec. V).

It is important to notice that the domains where the NIBA is justified are not easily established $a$ priori. Rather, one should verify self-consistently at the end of the calculation that the mean blip and sojourn lengths obey the condition $\langle\tau\rangle \ll\langle\rho\rangle$. The NIBA consists of the two simplifying prescriptions: (i) Set all factors $X_{j, k}$ in (3.9) equal to zero for $j \neq k+1$, and put $X_{k+1, k}=R\left(\tau_{k+1}\right)$. Hence in this approximation, $\varphi_{k, n}=\xi_{k+1} R\left(\tau_{k+1}\right)$. (ii) Set all the interblip interactions $\Lambda_{j, k}$ (3.9) in the factor $Q_{n}$ equal to zero.

With (i) and (ii), it is straightforward to sum over all possible blip configurations in given order $n$. This yields

$$
\begin{aligned}
\widehat{P}(\lambda)= & \frac{1}{\lambda}+\frac{1}{\lambda} \sum_{n=1}^{\infty}\left(-\Delta^{2}\right)^{n} \\
& \times \int_{0}^{\infty} \widetilde{\mathcal{D}}_{n}(\lambda) e^{-S\left(\tau_{1}\right)} \cos \left[\phi_{1}+R\left(\tau_{1}\right)\right] \\
& \times \prod_{k=2}^{n} \cos R\left(\tau_{k}\right) \cos \left(\phi_{k}\right) e^{-S\left(\tau_{k}\right)}
\end{aligned}
$$

In the absence of driving, the integrand is factorized in all the variables $\left\{\rho_{j}\right\}$ and $\left\{\tau_{j}\right\}$, and the sum is in the form of a geometrical series, which is easily summed. For timedependent driving, the situation is considerably complicated, since the phase $\phi_{k}^{(1)}$ associated with the $k$ th blip depends on all the previous sojourns and blip lengths. Phrasing differently, the phase associated with a blip depends on the full past history of the system's path. As a consequence, the integrand in (3.24) no longer factorizes into contributions of the individual blip and sojourn intervals. This complexity prevents us from performing a straightforward analytic resummation.

For low driving frequencies, the system makes many transitions within a period. Then, under condition (3.21), we may linearize the phase $\phi_{k}^{(1)}$ in the blip lengths $\tau_{j}$. Under this approximation, an analytic resummation of (3.24) is possible. This will be discussed in Sec. V. For higher driving frequencies, a similar simplification is not possible. In order to tackle the general case, it is convenient to introduce

$$
\begin{aligned}
F_{\lambda}(t):= & \Delta^{2} \int_{0}^{\infty} d \tau e^{-\lambda \tau-S(\tau)} \\
& \times \sin R(\tau) \sin \left[\epsilon_{0} \tau+g(t+\tau)-g(t)\right] \\
G_{\lambda}(t):= & \Delta^{2} \int_{0}^{\infty} d \tau e^{-\lambda \tau-S(\tau)} \\
& \times \cos R(\tau) \cos \left[\epsilon_{0} \tau+g(t+\tau)-g(t)\right] .
\end{aligned}
$$

These functions occur from integration over a blip length, and we have divided them into contributions that are odd and even in the bias, respectively. Here, the time $t$ is the sum of all blip and sojourn lengths preceding the respective blip. For periodic driving, the functions $F_{\lambda}(t)$ and $G_{\lambda}(t)$ are periodic as well, and therefore it is convenient to expand them in Fourier series,

$$
\begin{gathered}
F_{\lambda}(t)=\sum_{m=-\infty}^{\infty} f_{m}(\lambda) e^{-i m \omega t}, \\
G_{\lambda}(t)=\sum_{m=-\infty}^{\infty} g_{m}(\lambda) e^{-i m \omega t},
\end{gathered}
$$

where $\omega$ is the fundamental frequency. Upon inserting these expansions into the series (3.24), all the integrals over the blip and sojourn lengths can be carried out. 
It is then straightforward to see that the ensuing series expression can be iteratively generated by using recursion relations. It is also immediately clear from (3.26) that the coefficients $g_{0}(\lambda)$ that appear in the series expression do not depend on the history of the respective preceding blip and sojourn lengths. As a consequence of this, they appear as involved geometrical series expansions, which can be summed explicitly. Proceeding along these lines, we find

$$
\widehat{P}(\lambda)=\frac{1}{\lambda+g_{0}(\lambda)} \sum_{j=0}^{\infty} K_{j}(\lambda),
$$

where the functions $K_{j}(\lambda)$ are recursively determined by the formula

$$
K_{j+1}(\lambda)=\sum_{m \neq 0} \frac{-g_{m}(\lambda)}{\lambda+i m \omega+g_{0}(\lambda+i m \omega)} K_{j}(\lambda+i m \omega)
$$

with the starting expression

$$
K_{0}(\lambda)=1+\sum_{m^{\prime}} \frac{f_{m^{\prime}}(\lambda)}{\lambda+i m^{\prime} \omega}
$$

Equation (3.27) with (3.28) and (3.29) represents the formal solution of the problem in the presence of arbitrarily strong and arbitrarily fast time-periodic external driving. Here we have taken into account the quantum-stochastic forces related to the eliminated heat bath within the NIBA.

\section{Linear response limit}

On the assumption that the driving force is weak, Eq. (3.27) is evaluated further by linearizing it in the amplitude $\hat{\epsilon}$ of the time-dependent force. To this, we define the zero order functions,

$$
\begin{aligned}
& u\left(\lambda, \epsilon_{0}\right):=\Delta^{2} \int_{0}^{\infty} d \tau e^{-\lambda \tau-S(\tau)} \sin R(\tau) \sin \left(\epsilon_{0} \tau\right) \\
& v\left(\lambda, \epsilon_{0}\right):=\Delta^{2} \int_{0}^{\infty} d \tau e^{-\lambda \tau-S(\tau)} \cos R(\tau) \cos \left(\epsilon_{0} \tau\right)
\end{aligned}
$$

For notational simplification, we omit the parameter $\epsilon_{0}$ in the arguments of these functions in the remainder of this $\left[u\left(\lambda, \epsilon_{0}\right)=u(\lambda)\right.$, etc.]. The functions to linear order in the driving amplitude $\hat{\epsilon}$ are given by

$$
\begin{aligned}
F_{\lambda}^{(1)}(t):= & \Delta^{2} \int_{0}^{\infty} d \tau e^{-\lambda \tau-S(\tau)} \\
& \times \sin R(\tau) \cos \left(\epsilon_{0} \tau\right)[g(\tau+t)-g(t)] \\
= & \sum_{m=-\infty}^{\infty} f_{m}^{(1)}(\lambda) e^{-i m \omega t}, \\
G_{\lambda}^{(1)}(t):= & \Delta^{2} \int_{0}^{\infty} d \tau e^{-\lambda \tau-S(\tau)} \\
& \times \cos R(\tau) \sin \left(\epsilon_{0} \tau\right)[g(\tau+t)-g(t)] \\
= & \sum_{m=-\infty}^{\infty} g_{m}^{(1)}(\lambda) e^{-i m \omega t} .
\end{aligned}
$$

Keeping terms up to first order in $\hat{\epsilon}$, we find from (3.27)-(3.29)

$$
\widehat{P}(\lambda)=\widehat{P}^{(0)}(\lambda)+\widehat{P}^{(1)}(\lambda),
$$

where $\widehat{P}^{(0)}(\lambda)$ is the standard NIBA result for a static bias

$$
\widehat{P}^{(0)}(\lambda)=\frac{1+u(\lambda) / \lambda}{\lambda+v(\lambda)}
$$

and where the solution to first order in the driving amplitude is given by

$$
\begin{aligned}
\widehat{P}^{(1)}(\lambda)= & \frac{1}{\lambda+v(\lambda)} \sum_{m \neq 0} \frac{-g_{m}^{(1)}(\lambda)}{\lambda+i m \omega+v(\lambda+i m \omega)} \\
& +\frac{1}{\lambda+v(\lambda)} \sum_{m \neq 0} \frac{1}{\lambda+i m \omega} \\
& \times\left(f_{m}^{(1)}(\lambda)-\frac{g_{m}^{(1)}(\lambda) u(\lambda+i m \omega)}{\lambda+i m \omega+v(\lambda+i m \omega)}\right) .
\end{aligned}
$$

The real or complex conjugate poles of $\widehat{P}^{(0)}(\lambda)$ determine the transient dynamics of the undriven TLS $[2,29]$ and they set the time scale for $P(t)$ on which it reaches the thermal equilibrium value, which in the NIBA takes the form $P_{\mathrm{eq}}=u(0) / v(0)$.

The linear-response result (3.34) provides already useful insight into the dynamics of the driven TLS. The time-periodic asymptotic behavior of this contribution is controlled by the purely imaginary poles at $\lambda=-i m \omega$. Thus at long times, $P^{(1)}(t)$ aquires the periodicity of the driving force. The time scale on which the transient behavior dies out is set by the complex conjugate solutions of the pole condition $\lambda+i m \omega+v(\lambda+i m \omega)=0$. Now, these solutions are given by $\lambda_{m}=\lambda_{0}-i m \omega$, where $\lambda_{0}$ are real or complex conjugate solutions of the pole condition $\lambda+v(\lambda)=0$ of the respective system in the absence of driving. Thus, the parameter regime in which the NIBA is justified is essentially the same as for the undriven system $[2,29]$. In the Ohmic case, the NIBA is justified for arbitrary temperature and bias when $\alpha>1$. For $\alpha \ll 1$, NIBA is valid when the condition $2 \pi k T \gg \hbar\left|\lambda+i \epsilon_{0}\right|$ is met, which roughly corresponds to the condition $k T \gg \hbar\left(\Delta^{2}+\epsilon_{0}^{2}\right)^{1 / 2}[29]$. Besides, the NIBA is also justified for arbitrary damping if the static bias $\epsilon_{0}$ is sufficiently effective in reducing the blip lengths. For weak damping, this means $\epsilon_{0} \gg \Delta$.

For monochromatic driving, only the $m= \pm 1$ terms in (3.34) contribute to $\widehat{P}^{(1)}(\lambda)$. Thus, in the asymptotic state, $P(t)=P^{(\text {as })}(t)$ oscillates coherently with the driving frequency $\omega$, and there are no higher harmonics in the linear-response limit. Observing that $P^{(\text {as })}(t)$ is related to the linear dynamical susceptibility $\chi(\omega)$ by

$$
P^{(\mathbf{a s})}(t)=\hbar \hat{\epsilon}\left[\chi(-\omega) e^{i \omega t}+\chi(\omega) e^{-i \omega t}\right],
$$

we find from (3.34) for the linear susceptibility 


$$
\begin{aligned}
\chi(\omega)= & \frac{1}{\hbar \hat{\epsilon}[-i \omega+v(-i \omega)]} \\
& \times\left(f_{1}^{(1)}(-i \omega)-g_{1}^{(1)}(-i \omega) \frac{u(0)}{v(0)}\right) .
\end{aligned}
$$

The real and imaginary part of the dynamical susceptibility exhibit the dispersive and absorptive properties of the TLS, as they become manifest in spectroscopy experiments with electromagnetic or elastic waves.

\section{Nonlinear response}

From the recursive form (3.27)-(3.29), we can extract a number of general features of the dynamics of the nonlinearly driven system.

Information about the transient behavior at intermediate times is gained from a study of the pole condition

$$
\lambda+i m \omega+g_{0}(\lambda+i m \omega)=0 .
$$

Because of the influence kernel $e^{-S(\tau)} \cos R(\tau)$ implicit in the integrand of $g_{0}(\lambda)$, the primary solutions $\lambda_{0}$, i.e., the solutions for $m=0$, are either negative real, or they appear as complex conjugate pairs with $\operatorname{Re} \lambda_{0}<0$. Hence they render exponential relaxation or damped oscillations. All the other solution for $m= \pm 1, \pm 2, \ldots$, are obtained by periodic shifts along the imaginary axes, $\lambda_{m}=\lambda_{0}-i m \omega$. The inverse of the time scale on which the transient behavior dies out is set by the smallest real part of these solutions.

The pure oscillatory behavior reached at long times is determined by the poles of the second term in (3.29). Since the residua of these poles are odd in $\varepsilon(t)$, it is directly clear that they do not give contributions to $P^{(s)}(t)$. This proves our earlier remark that $P^{(s)}(t)$ describes transient behavior only.

Upon picking up all poles stringed along the imaginary axis by Laplace contour integration, the asymptotic solution takes the form of a Fourier series

$$
P^{(\text {as) }}(t)=\sum_{m=-\infty}^{\infty} P_{m} e^{-i m \omega t} .
$$

Using (3.27) - (3.29), the Fourier coefficients are obtained in the form

$$
P_{m}=\frac{1}{-i m \omega+g_{0}(-i m \omega)} \sum_{j=0}^{\infty} H_{j}(m)
$$

where the functions $H_{j}(m)$ are iteratively calculated from the recursion relation

$$
\begin{aligned}
H_{j+1}(m)= & \sum_{m^{\prime} \neq 0} \frac{-g_{m^{\prime}}(-i m \omega)}{i\left(m^{\prime}-m\right) \omega+g_{0}\left[i\left(m^{\prime}-m\right) \omega\right]} \\
& \times H_{j}\left(m-m^{\prime}\right)
\end{aligned}
$$

with the starting value

$$
H_{0}(m)=f_{m}(-i m \omega) .
$$

It is also straightforward to derive from the formal solution a system of coupled equations for the Fourier coefficients $P_{m}$. We find

$$
P_{0}=\frac{f_{0}(0)}{g_{0}(0)}-\sum_{m \neq 0} \frac{g_{-m}(0)}{g_{0}(0)} P_{m}
$$

and for $m \neq 0$

$$
P_{m}=\frac{i}{m \omega}\left(f_{m}(-i m \omega)-\sum_{m^{\prime}} g_{m-m^{\prime}}(-i m \omega) P_{m^{\prime}}\right) \text {. }
$$

These are the central results of this work.

Before entering into a detailed study, some general features can easily be drawn from (3.38)-(3.40). Consider a system in which the static detuning energy $\epsilon_{0}$ is zero. It then follows from the defining expressions in (3.25) by general properties of Fourier series that the Fourier coefficients of $F_{\lambda}(t)$ with even index and the Fourier coefficients of $G_{\lambda}(t)$ with odd index vanish, i.e., $f_{2 m}(\lambda)=0$ and $g_{2 m+1}(\lambda)=0$. With this, it is straightforward to see from (3.38)-(3.40) that all Fourier coefficients of $P^{(\text {as })}(t)$ with even index vanish, $P_{2 m}=0, m=0, \pm 1, \pm 2, \ldots$. The same selection rule is known to hold in the so-called stochastic resonance phenomena of classical symmetric systems [23]. Our general solution shows that these features hold true also in the full quantum regime irrespective of the type of damping and the strength and spectral properties of the periodic driving force.

In the limit of linear response and monochromatic driving, the $m= \pm 1$ components are the only nonvanishing harmonics in the time-periodic asymptotic state. For increasing strength of the amplitude $\hat{\epsilon}$, higher harmonics get increasingly important. However, in a wide range of experimental setups, e.g., when lock-in techniques are used, or when the system of interest is coupled to a highquality macroscopic oscillator such as a resonant cavity or a vibrating reed [19], the principal mode $P_{ \pm 1}$ is usually the quantity that dominates the physics even for large $\hat{\epsilon}$. The principal mode is also of central interest in investigations of stochastic resonance phenomena in quantum systems [22-24]. Therefore, we shall primarily focus on the fundamental harmonics of $P(t)$ in describing the time-periodic asymptotic state.

\section{HIGH-FREQUENCY DRIVING}

In the high-frequency regime

$$
\omega \gg v(0),
$$

where $v(0)$ is defined in (3.30), both the transient and the periodic dynamics may be calculated from (3.27) and (3.41) with (3.42) in a suitable approximation. To derive the appropriate description of the transient dynamics in the regime (4.1), it is convenient to rewrite (3.27) in the form

$$
\widehat{P}(\lambda)=\frac{1+f_{0}(\lambda) / \lambda}{\lambda+g_{0}(\lambda)}+\frac{\sum_{j=0}^{\infty} K_{j}(\lambda)}{\lambda+g_{0}(\lambda)}
$$

where the functions $K_{j}(\lambda)$ are again determined recursively from (3.28) but with the starting value 


$$
\begin{aligned}
K_{0}(\lambda)= & \sum_{m \neq 0}\left[\frac{f_{m}(\lambda)}{\lambda+i m \omega}-\frac{g_{m}(\lambda)}{\lambda+i m \omega+g_{0}(\lambda+i m \omega)}\right. \\
& \left.\times\left(1+\frac{f_{0}(\lambda+i m \omega)}{\lambda+i m \omega}\right)\right]
\end{aligned}
$$

From this we see that the second term in (4.2) contains at least a factor $g_{m}\left(\lambda_{0}+i n \omega\right) / \omega$ or $f_{m}\left(\lambda_{0}+i n \omega\right) / \omega$, and in each step of the iteration an additional factor $g_{m}\left(\lambda_{0}+i n \omega\right) / \omega$ is involved. Here, as discussed in the previous section, $\lambda_{0}$ is a complex number with $\operatorname{Re} \lambda_{0} \leq 0$. As we shall see shortly, the coefficients $g_{m}, f_{m}$ obey the condition $\left|g_{m}\left(\lambda_{0}+i n \omega\right)\right|<v(0),\left|f_{m}\left(\lambda_{0}+i n \omega\right)\right|<$ $P_{\text {eq }} v(0)<v(0)$ for all $m, n$. Hence the ensuing series is rapidly converging when the condition (4.1) is met. Here, $P_{\text {eq }}=u(0) / v(0)$ is the static equilibrium value within NIBA and $u(0), v(0)$ are defined in (3.30). The case of Ohmic damping is discussed in some detail in Appendix A where the function $v(0)$ is explicitly evaluated. In the high-frequency domain (4.1), the transient dynamics is then fairly well described by the first term in (4.2)

$$
\widehat{P}^{(\operatorname{tr})}(\lambda)=\frac{1+f_{0}(\lambda) / \lambda}{\lambda+g_{0}(\lambda)}
$$

The inverse time scale on which the system relaxes to the asymptotic state is given by the zeros of $\lambda+g_{0}(\lambda)$.

In the time-periodic asymptotic regime, the Fourier coefficients $P_{m}$ are conveniently calculated by iteration of (3.42). To leading order in $\left|g_{m}(i n \omega)\right| / \omega \ll 1$, we then find for the asymptotic state

$$
\begin{aligned}
P^{(\mathrm{as})}(t) & =\frac{f_{0}(0)}{g_{0}(0)}+\sum_{m \neq 0} P_{m}(\omega) e^{-i m \omega t} \\
P_{m}(\omega) & =\frac{i}{m \omega}\left(f_{m}(-i m \omega)-g_{m}(-i m \omega) \frac{f_{0}(0)}{g_{0}(0)}\right) .
\end{aligned}
$$

It should be noted that the quasiequilibrium value $P_{\mathrm{eq}}^{(\text {as })}=f_{0}(0) / g_{0}(0)$, which is the time average of $P^{(\text {as })}(t)$, differs from the equilibrium of the static case within the NIBA $P_{\text {eq }}=u(0) / v(0)$. Only in the limit where the system's frequency scales are small compared with the driving frequency $\omega$, the two quantities coincide. We also see from (4.5) that, as a result of the system's inertia, the time-dependent part drops to zero inversely with the driving frequency.

For monochromatic driving, the Fourier coefficient functions $f_{m}(\lambda)$ and $g_{m}(\lambda)$ take the form

$$
\begin{aligned}
& f_{2 k}(\lambda)=(-1)^{k} \Delta^{2} \int_{0}^{\infty} d \tau e^{-\lambda \tau-S(\tau)} e^{-i k \omega \tau} \\
& \quad \times \sin R(\tau) \sin \left(\epsilon_{0} \tau\right) J_{2 k}\left(\frac{2 \hat{\epsilon}}{\omega} \sin \frac{\omega \tau}{2}\right), \\
& f_{2 k+1}(\lambda)=(-1)^{k} \Delta^{2} \int_{0}^{\infty} d \tau e^{-\lambda \tau-S(\tau)} e^{-i(k+1 / 2) \omega \tau} \\
& \quad \times \sin R(\tau) \cos \left(\epsilon_{0} \tau\right) J_{|2 k+1|}\left(\frac{2 \hat{\epsilon}}{\omega} \sin \frac{\omega \tau}{2}\right),
\end{aligned}
$$

and

$$
\begin{aligned}
g_{2 k}(\lambda)= & (-1)^{k} \Delta^{2} \int_{0}^{\infty} d \tau e^{-\lambda \tau-S(\tau)} e^{-i k \omega \tau} \\
& \times \cos R(\tau) \cos \left(\epsilon_{0} \tau\right) J_{2 k}\left(\frac{2 \hat{\epsilon}}{\omega} \sin \frac{\omega \tau}{2}\right), \\
g_{2 k+1}(\lambda)= & (-1)^{k+1} \Delta^{2} \int_{0}^{\infty} d \tau e^{-\lambda \tau-S(\tau)} e^{-i(k+1 / 2) \omega \tau} \\
& \times \cos R(\tau) \sin \left(\epsilon_{0} \tau\right) J_{|2 k+1|}\left(\frac{2 \hat{\epsilon}}{\omega} \sin \frac{\omega \tau}{2}\right)
\end{aligned}
$$

where $J_{m}(z)$ is a Bessel function of the first kind. Upon setting $\epsilon_{0}=0$ and inserting the respective form for the coefficient $g_{0}(\lambda)$ into Eq. (4.4), the solution by Dakhnovskii [16] in the transient regime is recovered. Now, upon using the form (4.6) for $f_{0}(\lambda)$ in (4.4), we obtain the respective generalization to the biased case. Similarly, knowledge about the asymptotic behavior may be obtained by inserting (4.6) and (4.7) into (4.5).

Since $\left|J_{m}(z)\right| \leq 1$ for all $m$ and $z$ and $\operatorname{Re} \lambda<0$, the previously mentioned condition $\left|g_{m}(\lambda+i n \omega)\right|<v(0)$ is satisfied for all $m, n$, and $\lambda$. From this we see that in addition to the interblip correlation factor $e^{-S(\tau)}$ originating from the stochastic force, the oscillating driving field is also effective in reducing the blip lengths. Thus, whenever the NIBA is applicable in the absence of the time-periodic field (see the previous discussion and Refs. $[2,9])$, it is justified even better in the presence of highfrequency driving. The Ohmic case is discussed in some detail in Appendix A.

The analysis of the expressions (4.6) and (4.7) in the regime $\omega \gg\left|\epsilon_{0}\right|$ given in Appendix B provides important insights into the physics of the system under consideration. Upon using the forms (B1) and (B2), we see that all the Fourier coefficients $P_{m}(\omega)$ are zero when $\hat{\epsilon} / \omega$ is a zero of $J_{0}(\hat{\epsilon} / \omega)$. This condition resembles the one leading to the phenomenon of coherent destruction of tunneling discussed in Refs. [12-14]. In addition, for $n \neq 0$, an individual coefficient $P_{n}$ vanishes when $\hat{\epsilon} / \omega$ is a zero of $J_{n}(\hat{\epsilon} / \omega)$.

In the frequency range below $\epsilon_{0}$, we are dependent on

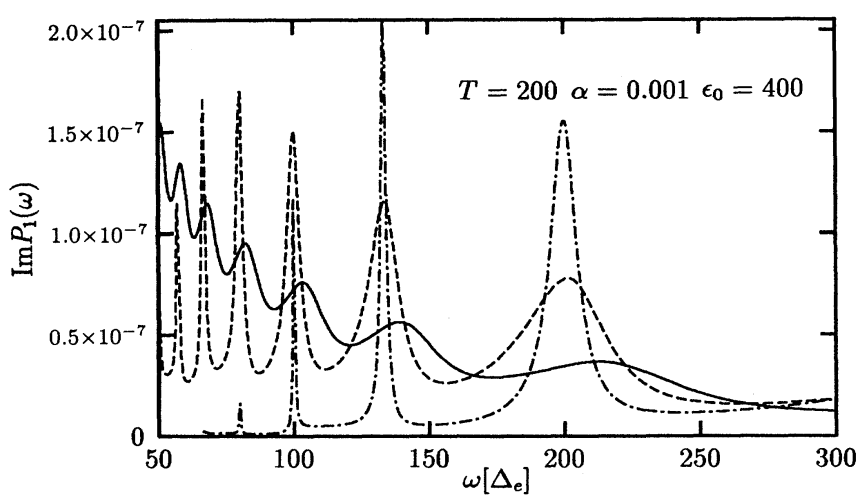

FIG. 1. Imaginary part of the fundamental harmonic $P_{1}(\omega, \hat{\epsilon})$ as a function of the driving frequency for different values of the driving amplitude. We have chosen $\hat{\epsilon}=$ $100 \Delta, 200 \Delta$, and $400 \Delta$ (dashed-dotted, dashed, and full line, respectively). 


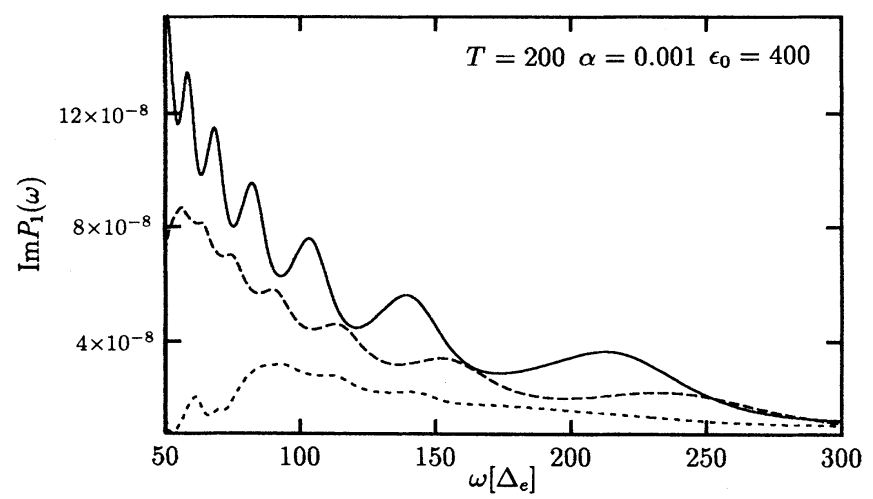

FIG. 2. Same plot with same values for $\alpha, T$, and $\epsilon_{0}$ as in Fig. 1, but with different values of the driving amplitude, $\hat{\epsilon}=400 \Delta, 600 \Delta$, and $1000 \Delta$ (full, dashed, and dotted line, respectively).

a numerical calculation. We have performed a numerical analysis of the fundamental harmonic in the nonlinearresponse regime for weak Ohmic dissipation $\alpha \ll 1$ and high temperature $k T \gg \hbar \Delta$. The Fourier coefficient $P_{1}(\omega)$ exhibits pronounced resonances as a function of the driving frequency. Figures 1 and 2 show plots of $\operatorname{Im} P_{1}(\omega)$ for $\alpha=0.001, k T=200 \hbar \Delta$, and $\epsilon_{0}=400 \hbar \Delta$ for different values of the driving amplitude. At the lowest chosen value $\hat{\epsilon}=100 \Delta$ (dashed-dotted curve in Fig. 1), the resonances appear at $\omega=\epsilon_{0}$ and at fractional values of the static bias, $\omega=\omega_{n}$ with $\omega_{n}=\epsilon_{0} / n$, $(n=2,3,4, \ldots$,$) . With increasing \hat{\epsilon}$, the resonances are decreasingly marked, and their positions are shifted to slightly higher frequencies. For $\hat{\epsilon}=400 \Delta$ and $\hat{\epsilon}=600 \Delta$, the envelope function is a smooth function peaked near zero frequency (see Fig. 2).

\section{LOW-FREQUENCY DRIVING}

When the driving frequency $\omega$ is low enough that the condition

$$
\omega\langle\tau\rangle \ll 1
$$

is met, an analytic resummation of the series (3.24) is possible. Under condition (3.21), we may linearize the phase $\phi_{j}^{(1)}$ in (3.20) in the blip lengths $\tau_{j}$, yielding for the total phase $\Phi_{n}$ in (3.19) the form

$$
\Phi_{n}^{(\ell)}=\sum_{j=1}^{n}\left[\epsilon_{0}+\hat{\epsilon} f\left(\sum_{i=1}^{j} \rho_{i}\right)\right] \xi_{j} \tau_{j} .
$$

In this approximation, the breathing-mode integrals (3.25) take the form

$$
\begin{aligned}
F_{\lambda}^{(\ell)}(\rho):= & \Delta^{2} \int_{0}^{\infty} d \tau e^{-\lambda \tau-S(\tau)} \\
& \times \sin R(\tau) \sin \left\{\left[\epsilon_{0}+\hat{\epsilon} f(\rho)\right] \tau\right\} \\
G_{\lambda}^{(\ell)}(\rho):= & \Delta^{2} \int_{0}^{\infty} d \tau e^{-\lambda \tau-S(\tau)} \\
& \times \cos R(\tau) \cos \left\{\left[\epsilon_{0}+\hat{\epsilon} f(\rho)\right] \tau\right\}
\end{aligned}
$$

Here for an individual blip, $\rho$ is the sum of the lengths of all the preceding sojourns. Upon using these linearized expressions in (3.24), the symmetric and antisymmetric parts of the series can be summed to the form [3]

$$
\begin{aligned}
\widehat{P}^{(a)}(\lambda)= & \int_{0}^{\infty} d \rho_{0} F_{\lambda}^{(\ell)}\left(\rho_{0}\right) e^{-\lambda \rho_{0}} \int_{0}^{\infty} d \rho_{1} e^{-\lambda \rho_{1}} \\
& \times \exp \left(-\int_{\rho_{0}}^{\rho_{0}+\rho_{1}} d \rho G_{\lambda}^{(\ell)}(\rho)\right), \\
\widehat{P}^{(s)}(\lambda)= & \int_{0}^{\infty} d \rho_{0} e^{-\lambda \rho_{0}} \exp \left(-\int_{0}^{\rho_{0}} d \rho G_{\lambda}^{(\ell)}(\rho)\right) .
\end{aligned}
$$

Let us next consider the important case where the dependence on $\lambda$ in the functions $F_{\lambda}^{(\ell)}(\rho)$ and $G_{\lambda}^{(\ell)}(\rho)$ may be neglected. This is possible in the parameter regime where $|\varepsilon| \gg \Delta$ for any type of damping, or in the Ohmic case for strong damping $\alpha>1$ and any temperature and bias, or for weak damping $\alpha \ll 1$, when the temperature is sufficiently high, $k T>\hbar \Delta / \pi \alpha$ (see, e.g., Ref. [9]). Then the inverse Laplace transform of (5.4) can be performed exactly and we find for the two contributions in (3.15)

$$
\begin{aligned}
& P^{(a)}(t)=\int_{0}^{t} d \rho F_{0}^{(\ell)}(\rho) \exp \left(-\int_{\rho}^{t} d \rho_{0} G_{0}^{(\ell)}\left(\rho_{0}\right)\right) \\
& P^{(s)}(t)=\exp \left(-\int_{0}^{t} d \rho G_{0}^{(\ell)}(\rho)\right)
\end{aligned}
$$

It is straightforward to verify that upon using these forms $P(t)$ obeys the first order differential equation

$$
\dot{P}(t)=-G_{0}^{(\ell)}(t)\left[P(t)-\bar{P}_{\text {eq }}(t)\right],
$$

which is in the form of a rate equation with a timedependent rate and the time-dependent adiabatic equilibrium value $\bar{P}_{\text {eq }}(t):=F_{0}^{(\ell)}(t) / G_{0}^{(\ell)}(t)$.

Let us finally discuss the asymptotic state of the general case. Noting that the functions $F_{\lambda}^{(\ell)}(\rho)$ and $G_{\lambda}^{(\ell)}(\rho)$ are periodic functions of $\rho$ with period $2 \pi / \omega$, we can map the interval $[0, \infty]$ of the $\rho_{0}$ integral in $(5.4)$ on the principal interval $[0,2 \pi / \omega]$. We then find

$$
\widehat{P}^{(a)}(\lambda)=\frac{K(\lambda, \lambda)}{1-e^{-2 \pi \lambda / \omega}},
$$

where the function $K(\lambda, \mu)$ is given by

$$
\begin{aligned}
K(\lambda, \mu)= & \int_{0}^{2 \pi / \omega} d \rho_{0} F_{\mu}^{(\ell)}\left(\rho_{0}\right) e^{-\lambda \rho_{0}} \\
& \times \int_{0}^{\infty} d \rho_{1} e^{-\lambda \rho_{1}} \exp \left(-\int_{\rho_{0}}^{\rho_{0}+\rho_{1}} d \rho G_{\mu}^{(\ell)}(\rho)\right)
\end{aligned}
$$

By taking into account in the inverse Laplace transformation the zeros of the denominator in (5.7), we obtain the asymptotic state in the form of a Fourier series,

$$
\begin{aligned}
P^{(\mathrm{as})}(t) & =\sum_{m=-\infty}^{\infty} P_{m}(\omega) e^{-i m \omega t} \\
P_{m} & =\frac{\omega}{2 \pi} K(-i m \omega,-i m \omega) .
\end{aligned}
$$


The behavior of the fundamental harmonic $P_{1}(\omega)$ in the frequency range (5.1) has been discussed in previous work [3]. For weak Ohmic damping, the function $P_{1}(\omega)$ does not exhibit a structure as rich as seen in Figs. 1 and 2 in the high-frequency regime. Rather one finds that $\operatorname{Im} P_{1}(\omega)$ has a maximum near $\omega=10^{-3}-10^{-5} \Delta$ depending on the parameters, and the slopes to the left and right of the maximum away from a narrow rounding region turn out to be constant in a double-logarithmic plot.

We conclude this section with the remark that if we put approximately $P_{m}(\omega)=(\omega / 2 \pi) K(-i m \omega, 0)$, the Fourier series (5.9) can be summed in analytic form. It is just this way in which we recover the previous expression (5.5) for $P^{(a)}(t)$.

\section{CONCLUSIONS}

In this work, we have studied the dynamics of the periodically driven spin-boson system. By use of a realtime path integral description, we have investigated both the transient and the time-periodic asymptotic dynamics within the noninteracting-blip approximation for the stochastic forces. We have obtained the solution for any strength, shape in time, and period of the driving force in the form of a series whose terms are defined by recursion relations as given in (3.27)-(3.29). We also have found expressions in analytic form in the limiting cases of low and high frequencies. In contrast to the static case, driving-induced correlations between all of the system's transitions render the dynamics intrinsically nonMarkovian. Only at low frequencies of the driving force, we recover a regime where the dynamics is described by a simple rate equation with a time-dependent rate. For high-frequency driving, resonance phenomena, such as resonances near fractional values of the intrinsic static bias are observed for the ubiquitous case of Ohmic friction and monochromatic driving.

Our results are valid independently of the specific time dependence of the force profile and the specific dissipative mechanism. Therefore, they might be useful to describe a plethora of phenomena such as suppression of tunneling $[5,12,14]$, anomalous low-frequency generation and high harmonic generation [7], nonlinear low-temperature response in ac-perturbed metallic glasses [17-19], and quantum stochastic resonance [24]. Since we did neither invoke a Markovian nor a rotating wave approximation, our formalism is also useful in the vast field of "quantum control" of physical and chemical processes as they emerge from tailored femtosecond pulse sequences $[6,30$, $31]$.

\section{ACKNOWLEDGMENTS}

We gratefully acknowledge partial financial support by the Volkswagenstiftung under "Resonance Activation" Contract No. Az I/65037, the Deutsche Forschungsgemeinschaft (DFG, Bonn) under Contract No. Ha $1517 / 14-1$, and by the SCIENCE programme of the European Community under Contract No. SCI*-CT91-0656 (TSTS).

\section{APPENDIX A: OHMIC DISSIPATION}

Within the NIBA, the effects of the environment are in the integrals over the blip lengths. Upon inserting the Ohmic form (3.22) in (3.30), we find

$$
v(\lambda, 0)=\frac{\Delta_{e}}{\pi}\left(\frac{\hbar \beta \Delta_{e}}{2 \pi}\right)^{1-2 \alpha} \frac{\Gamma(\alpha+\hbar \beta \lambda / 2 \pi)}{\Gamma(1-\alpha+\hbar \beta \lambda / 2 \pi)},
$$

where $\Gamma(z)$ is the gamma function and where we introduced the effective zero temperature tunneling splitting

$$
\Delta_{e}=\Delta\left(\frac{\Delta}{\omega_{c}}\right)^{\alpha /(1-\alpha)}[\cos (\pi \alpha) \Gamma(1-2 \alpha)]^{1 /(2-2 \alpha)}
$$

We then have

$$
\begin{aligned}
& v\left(\lambda, \epsilon_{0}\right)=\left[v\left(\lambda+i \epsilon_{0}, 0\right)+v\left(\lambda-i \epsilon_{0}, 0\right)\right] / 2 \\
& u\left(\lambda, \epsilon_{0}\right)=\frac{-\tan \pi \alpha}{2 i}\left[v\left(\lambda+i \epsilon_{0}, 0\right)-v\left(\lambda-i \epsilon_{0}, 0\right)\right] .
\end{aligned}
$$

For weak damping $\alpha \ll 1$ we get

$$
v\left(0, \epsilon_{0}\right)=\frac{\hbar \beta \Delta_{e}^{2}}{2 \pi} \frac{\alpha \hbar \beta \epsilon_{0} / 2}{\alpha^{2}+\left(\hbar \beta \epsilon_{0} / 2 \pi\right)^{2}} \operatorname{coth} \frac{\hbar \beta \epsilon_{0}}{2} .
$$

For high temperatures, we may use the expression (3.23) in the defining integral (3.30) for $v\left(\lambda, \epsilon_{0}\right)$. Then we find

$$
\begin{aligned}
v\left(\lambda, \epsilon_{0}\right)= & \Delta_{e}\left(\frac{\hbar \beta \Delta_{e}}{2 \pi}\right)^{1-2 \alpha} \frac{\Gamma(1+\alpha)}{\Gamma(1-\alpha)} \\
& \times \frac{\alpha+\beta \lambda / 2 \pi}{(\alpha+\beta \lambda / 2 \pi)^{2}+\left(\hbar \beta \epsilon_{0} / 2 \pi\right)^{2}} .
\end{aligned}
$$

Upon using these forms, it is easy to trace out the parameter regime in which the high-frequency limit $\omega \gg$ $v\left(0, \epsilon_{0}\right)$, which founded the basis of the discussion in Sec. IV, is valid.

As stated already in Sec. IV, when the NIBA is justified in the static case, it should hold even better in the presence of high-frequency forces. Using (A4) and (A5), this can be specified quantitatively. By virtue of the relation

$$
J_{0}[2 z \sin (a / 2)]=J_{0}^{2}(z)+2 \sum_{n=1}^{\infty} J_{n}^{2}(z) \cos n a,
$$

we may express the function $g_{0}(\lambda)$ in terms of the function $v\left(\lambda, \epsilon_{0}\right)$ involving static properties only,

$$
g_{0}(\lambda)=\sum_{n=-\infty}^{\infty} J_{n}^{2}(\hat{\epsilon} / \omega) v\left(\lambda, \epsilon_{0}+n \omega\right)
$$

There follows from (A4) and (A5) that the function $g_{0}(\lambda)$ has a maximum at $\epsilon_{0}=0$. From this we may conclude

(i) when $\hat{\epsilon} \leq \omega, \epsilon_{0}<\omega$, the term with $n=0$ dominates the series (A7). Hence the effect of the driving field is simply to renormalize the effective tunneling matrix ele- 
ment $\Delta_{e}$ in $(\mathrm{A} 2)$ as

$$
\Delta_{0}=\Delta_{e}\left|J_{0}(\hat{\epsilon} / \omega)\right|^{1 / 1-\alpha} .
$$

This allows for a simple criterion: The NIBA is justified in the presence of driving when it is justified in the undriven case for the effective tunnel splitting $\Delta_{0}$.

(ii) When $\hat{\epsilon} \leq \omega, \epsilon_{0} \geq \omega$, the series of Bessel functions is still dominated by the first term proportional to $J_{0}$ but the main contribution from the static functions arises when $n=k$, where $\left|\epsilon_{0}-k \omega\right|:=\delta<\omega$. In this case we may estimate

$$
\begin{aligned}
g_{0}(\lambda) \approx & J_{0}^{2}(\hat{\epsilon} / \omega) v\left(\lambda, \epsilon_{0}\right)+J_{k}^{2}(\hat{\epsilon} / \omega) v\left(\lambda, \epsilon_{0}-k \omega\right) \\
& +J_{k+1}^{2}(\hat{\epsilon} / \omega) v\left[\lambda, \epsilon_{0}-(k+1) \omega\right]
\end{aligned}
$$

In particular, when $\delta=0$, one has $g_{0}(\lambda) \approx$ $J_{0}^{2}(\hat{\epsilon} / \omega) v\left(\lambda, \epsilon_{0}\right)+J_{\epsilon_{0} / \omega}^{2}(\hat{\epsilon} / \omega) v(\lambda, 0)$. Depending on the relative importance of the two terms, the system effectively may behave as a biased system with splitting $\Delta_{0}$ or as a symmetric system with splitting $\Delta_{\epsilon_{0} / \omega}=$ $\Delta_{e}\left|J_{\epsilon_{0} / \omega}(\hat{\epsilon} / \omega)\right|^{1 / 1-\alpha}$.

In the opposite limit $\hat{\epsilon} / \omega \gg 1$, matters become more complicated since for arguments $z>k$ the Bessel functions behave qualitatively as sine or cosine functions with amplitudes that decay as $z^{-1 / 2}$. For $z \gg n$, we have asymptotically $J_{n}(z) \simeq \sqrt{2 / \pi z} \cos [z-(2 n+1) \pi / 4]$. In the strongly nonlinear regime $\hat{\epsilon} \gg \omega$, the series of Bessel functions is dominated by the terms with $n<\hat{\epsilon} / \omega$, which almost equally contribute. Thus, to estimate the validity of NIBA many terms of the series (A7) should be taken into account.

Finally, upon inserting (A1) and (A3) in (A7), it can be shown that $\operatorname{Re} g_{0}(\lambda)$ is nonzero for imaginary $\lambda$ so that the zeros of (3.36) contribute to the transient dynamics of the system only.

\section{APPENDIX B: SUPPRESSION OF HARMONICS}

When $\omega\langle\tau\rangle \gg 1$ and when the driving frequency $\omega$ is very large compared with the static bias $\epsilon_{0}$, it is convenient to utilize a separation of the appearing time scales in the expressions (4.6) and (4.7) for the coefficients $f_{n}(-i m \omega)$ and $g_{n}(-i m \omega)$. We then find

$$
\begin{aligned}
& f_{2 k}(-i m \omega)=(-1)^{m} J_{2 k-m}(\hat{\epsilon} / \omega) J_{m}(\hat{\epsilon} / \omega) u\left(0, \epsilon_{0}\right), \\
& f_{2 k+1}(-i m \omega)=i(-1)^{m} J_{2 k+1-m}(\hat{\epsilon} / \omega) J_{m}(\hat{\epsilon} / \omega) x\left(\epsilon_{0}\right),
\end{aligned}
$$

and

$$
\begin{aligned}
& g_{2 k}(-i m \omega)=(-1)^{m} J_{2 k-m}(\hat{\epsilon} / \omega) J_{m}(\hat{\epsilon} / \omega) v\left(0, \epsilon_{0}\right) \\
& , g_{2 k+1}(-i m \omega)=i(-1)^{m+1} J_{2 k+1-m}(\hat{\epsilon} / \omega) J_{m}(\hat{\epsilon} / \omega) y\left(\epsilon_{0}\right),
\end{aligned}
$$

where the functions $u\left(0, \epsilon_{0}\right)$ and $v\left(0, \epsilon_{0}\right)$ are defined in (3.30), and where

$$
\begin{aligned}
& x\left(\epsilon_{0}\right)=\Delta^{2} \int_{0}^{\infty} d \tau e^{-S(\tau)} \sin R(\tau) \cos \left(\epsilon_{0} \tau\right), \\
& y\left(\epsilon_{0}\right)=\Delta^{2} \int_{0}^{\infty} d \tau e^{-S(\tau)} \cos R(\tau) \sin \left(\epsilon_{0} \tau\right) .
\end{aligned}
$$

Thus the coefficients $f_{m}(-i m \omega)$ and $g_{m}(-i m \omega)$ are zero when $\hat{\epsilon} / \omega$ is either a zero of $J_{0}(\hat{\epsilon} / \omega)$ or a zero of $J_{m}(\hat{\epsilon} / \omega)$. As shown in Sec. IV, these properties are decisive for the behavior of the coefficients $P_{m}(\omega)$ of the asymptotic state specified in the Fourier series (4.5).
[1] R. Zwanzig, J. Stat. Phys. 9, 215 (1973); A. O. Caldeira and A. J. Leggett, Phys. Rev. Lett. 46, 211 (1981); Ann. Phys. (N.Y.) 149, 374 (1983); 153, 445(E) (1983).

[2] A. J. Leggett, S. Chakravarty, A. T. Dorsey, M. P. A. Fisher, A. Garg, and W. Zwerger, Rev. Mod. Phys. 59, 1 (1987).

[3] M. Grifoni, M. Sassetti, J. Stockburger, and U. Weiss, Phys. Rev. E 48, 3497 (1993).

[4] S. Chakravarty and S. Kivelson, Phys. Rev. Lett. 50, 1811 (1983); C. D. Tesche, Ann. N.Y. Acad. Sci. 480, 36 (1986).

[5] F. Grossman, Th. Dittrich, and P. Hänggi, Physica B 175, 293 (1991).

[6] J. E. Combariza, B. Just, J. Manz, and G. K. Paramonov, J. Phys. Chem. 95, 10351 (1992).

[7] R. Bavli and H. Metiu, Phys. Rev. Lett. 69, 1986 (1992); Yu Dakhnovskii and H. Metiu, Phys. Rev. A 47, 3299 (1993); 48, 2342 (1993).

[8] L. M. Sander and H. B. Shore, Phys. Rev. B 3, 1472 (1971).

[9] U. Weiss, Quantum Dissipative Systems, Series in Mod- ern Condensed Matter Physics Vol. 2 (World Scientific, Singapore, 1993).

[10] A. Benderskii, D. E. Makarov, and C. A. Wight, Adv. Chem. Phys. 88, 1 (1994).

[11] S. Chakravarty, Phys. Rev. Lett. 49, 681 (1982); A. J. Bray and M. A. Moore, ibid. 49, 1546 (1982); V. Hakim, A. Muramatsu, and F. Guinea, Phys. Rev. B 30, 464 (1984); A. Schmid, Phys. Rev. Lett. 51, 1506 (1983); S. Bulgadaev, Pis'ma Zh. Eksp. Teor. Fiz. 39, 264 (1984) [JETP Lett. 39, 314 (1985)]; F. Guinea, V. Hakim, and A. Muramatsu, Phys. Rev. Lett. 54, 263 (1985).

[12] F. Grossmann, P. Jung, Th. Dittrich, and P. Hänggi, Z. Phys. B 84, 315 (1991); Phys. Rev. Lett. 67, 516 (1991); M. Wagner, Phys. Rev. A 51, 798 (1995).

[13] F. Grossmann and P. Hänggi, Europhys. Lett. 18, 571 (1992).

[14] J. M. Gomez Llorente and J. Plata, Phys. Rev. A 45, R6958 (1992).

[15] Th. Dittrich, B. Oelschlägel, and P. Hänggi, Europhys. Lett. 22, 5 (1993).

[16] Yu Dakhnovskii, Phys. Rev. B 49, 4649 (1994); Ann. 
Phys. (N.Y.) 230, 145 (1994).

[17] J. Stockburger, M. Grifoni, M. Sassetti, and U. Weiss, Z. Phys. B 94, 447 (1994).

[18] P. Esquinazi, R. König, F. Pobell, F. Dietzel, G. Weiss, and S. Hunklinger, in Phonons 89 , edited by by S. Hunklinger, W. Ludwig, and G. Weiss (World Scientific, Singapore, 1990).

[19] P. Esquinazi, R. König, and F. Pobell, Z. Phys. B 87, 305 (1992).

[20] For a review of the standard tunneling model for metallic glasses, see J. L. Black, in Glassy Metals I, edited by H. J. Güntherodt and H. Beck, Topics in Applied Physics Vol. 46 (Springer, Berlin, 1981), Chap. 8.

[21] R. Benzi, G. Parisi, A. Sutera, and A. Vulpiani, Tellus 34, 10 (1982); F. Moss, A. Bulsara, and M. F. Shlesinger, J. Stat. Phys. 70, 1 (1993).

[22] B. McNamara and K. Wiesenfeld, Phys. Rev. A 39, 4854 (1989); P. Jung and P. Hänggi, Europhys. Lett. 8, 505 (1989).

[23] P. Jung and P. Hänggi, Phys. Rev. A 44, 8032 (1991).

[24] R. Löfstedt and S. N. Coppersmith, Phys. Rev. Lett. 72,
1947 (1994); Phys. Rev. E 49, 4821 (1994).

[25] J. Kondo, in Fermi Surface Effects, edited by J. Kondo and A. Yoshimori, Springer Series in Solid State Sciences Vol. 77 (Springer, Berlin, 1988).

[26] J. P. Sethna, Phys. Rev. B 24, 698 (1981); 25, 5050 (1982).

[27] A. K. Raychaudhuri and S. Hunklinger, Z. Phys. 57, 113 (1984); P. Esquinazi, H. M. Ritter, H. Neckel, G. Weiss, and S. Hunklinger, ibid. 64, 81 (1986).

[28] R. P. Feynman and F. L. Vernon, Ann. Phys. (N.Y.) 24, 118 (1963).

[29] U. Weiss and M. Wollensak, Phys. Rev. Lett. 62, 1663 (1989).

[30] D. J. Tannor and S. A. Rice, Adv. Chem. Phys. 70, 441 (1988); P. Brumer and M. Shapiro, Ann. Rev. Phys. Chem. 43, 257 (1992); W. S. Warren, H. Rabitz, and M. Dahleh, Science 259, 1581 (1993); J. Manz and L. Wöste, in Femtosecond Chemistry (VCH Publ., Weinheim, 1995).

[31] R. Kosloff, A. D. Hammerich, and D. J. Tannor, Phys. Rev. Lett. 69, 2172 (1992). 